\title{
sciendo
}

\author{
BULGARIAN ACADEMY OF SCIENCES
}

CYBERNETICS AND INFORMATION TECHNOLOGIES • Volume 20, No 6

Special Issue on New Developments in Scalable Computing

Sofia $\bullet 2020$

Print ISSN: 1311-9702; Online ISSN: 1314-4081

DOI: $10.2478 /$ cait-2020-0062

\section{A New Class of "Growth Functions" with Polynomial Variable Transfer Generated by Real Reaction Networks}

\section{Nikolay Kyurkchiev}

Faculty of Mathematics and Informatics, University of Plovdiv Paisii Hilendarski, 24 Tzar Asen Str., 4000 Plovdiv, Bulgaria

E-mail:nkyurk@uni-plovdiv.bg

Abstract: In [4, 5], two classes of growth models with "exponentially variable transfer" and "correcting amendments of Bateman-Gompertz-Makeham-type" based on a specific extended reaction network have been studied [1]. In this article we will look at the new scheme with "polynomial variable transfer". The consideration of such a dynamic model in the present article is dictated by our passionate desire to offer an adequate model with which to well approximate specific data in the field of computer viruses propagation, characterized by rapid growth in the initial time interval. Some numerical examples, using CAS Mathematica illustrating our results are given.

Keywords: Reaction networks, Generalized growth model, Exponentially and polynomial variable transfers.

\section{Introduction and preliminaries}

In [1] the following class of growth-decay model formulated in terms that include various types of evolution of the resource species:

$$
\begin{gathered}
S \stackrel{k_{1}}{\rightarrow} P \stackrel{k_{2}}{\rightarrow} Q, \\
k_{3} \stackrel{k_{4}}{\rightarrow} P_{1} \rightarrow Q_{1}, \\
\cdots \\
S \stackrel{k_{2 n-1}}{\rightarrow} P_{n-1} \stackrel{k_{2 n}}{\rightarrow} Q_{n-1}, \\
P_{n-1}+X \stackrel{k}{\rightarrow} n X+P_{n-1}
\end{gathered}
$$

is considered.

The general model can be written for the growth function in the form [1]:

$$
\left\{\begin{array}{c}
x^{\prime}(t)=k x P_{n-1}(t) \\
x(0)=x_{0}
\end{array}\right.
$$

where for $k_{i} \neq k_{j}, i, j=1,2, \ldots, 2 n$, 


$$
P_{n-1}(t)=\frac{k_{2 n-1} s_{0}}{k_{2 n}-\sum_{i=1}^{n} k_{2 i-1}}\left(e^{-\sum_{i=1}^{n} k_{2 i-1}}-e^{-k_{2 n^{t}}}\right)+p_{n-1,0} e^{-k_{2 n^{t}}} .
$$

We will explicitly note that for $n \geq 2$ the model (1)-(3) summarizes Markov's research $[2,3]$.

A number of basic results in this direction can be found in [6-11].

It is of interest to observe the new growth model based on this reaction networks in the case where, for example, $k_{1}=k_{1}(t), k_{3}=k_{3}(t), \ldots, k_{2 n-1}=k_{2 n-1}(t)$.

In this article we will get a generalized class of growth curves with polynomial variable transfer based on this reaction scheme.

\section{Main results}

Let us consider the following reaction network:

$$
\begin{gathered}
\stackrel{k_{1}(t)}{\rightarrow} P \stackrel{k_{2}}{\rightarrow} Q, \\
k_{3}(t) \stackrel{k_{4}}{\rightarrow} P_{1} \rightarrow Q_{1}, \\
\cdots \\
S \stackrel{k_{2 n-1}(t)}{\rightarrow} P_{n-1} \stackrel{k_{2 n}}{\rightarrow} Q_{n-1}, \\
P_{n-1}+X \stackrel{k}{\rightarrow} n X+P_{n-1},
\end{gathered}
$$

where

$$
k_{j}(t)=\sum_{i=1}^{n} a_{i}^{(j)} t^{i}, j=1,3, \ldots, 2 n-1,
$$

and $k_{r}, r=2,4, \ldots, 2 n$, and $k$ are constants.

In the general case, the detailed study of the scheme (4) and the corresponding "reaction system of differential equations", as well as the important question of the stability of the component of the solution $x(t)$ is an extremely difficult task.

For this reason, we will study the dynamics of this model in the following particular case.

2.1. The case $k_{1}(t)=1+t$, and $k_{2}=1$

Consequently the following differential equations can be formulated

$$
\left\{\begin{array}{l}
\frac{d s}{d t}=-(1+t) s, \\
\frac{d p}{d t}=(1+t) s-p, \\
\frac{d x}{d t}=k x p,
\end{array}\right.
$$


with $s(0)=s_{0} ; p(0)=p_{0} ; x(0)=x_{0}$.

For the solution of the first equation in (5) we obtain

$$
s(t)=C e^{-\left(t+\frac{1}{2} t^{2}\right)},
$$

and from $s(0)=s_{0}$ we get $C=s_{0}$ and

$$
s(t)=s_{0} e^{-\left(t+\frac{1}{2} t^{2}\right)} .
$$

From the second equation of the system (5) we have

$$
\frac{d p}{d t}+p(t)=s_{0}(1+t) e^{-\left(t+\frac{1}{2} t^{2}\right)} .
$$

The solution to this equation is

$$
\begin{aligned}
p(t) & =s_{0} e^{-t} \int_{0}^{t}(1+t) e^{-\left(t+\frac{1}{2} t^{2}\right)} e^{t} \cdot d t+R e^{-t}= \\
& =s_{0} e^{-t} \int_{0}^{t}(1+t) e^{-\frac{1}{2} t^{2}} \cdot d t+R e^{-t}= \\
& =\left.s_{0} e^{-t}\left(-e^{-\frac{1}{2} t^{2}}+\frac{\sqrt{\pi}}{2} \operatorname{Erf}\left(\frac{t}{\sqrt{2}}\right)\right)\right|_{0} ^{t}+R e^{-t}= \\
& =s_{0} e^{-t}\left(1-e^{-\frac{1}{2} t^{2}}+\frac{\sqrt{\pi}}{2} \operatorname{Erf}\left(\frac{t}{\sqrt{2}}\right)\right)+R e^{-t},
\end{aligned}
$$

where

$$
\operatorname{Erf}(z)=\frac{2}{\sqrt{\pi}} \int_{0}^{z} e^{-t^{2}} \cdot d t
$$

From $p(0)=p_{0}$ we get $R=p_{0}$.

So, finally, for the solution $p(t)$ we get

$$
p(t)=s_{0} e^{-t}\left(1-e^{-\frac{1}{2} t^{2}}+\frac{\sqrt{\pi}}{2} \operatorname{Erf}\left(\frac{t}{\sqrt{2}}\right)\right)+p_{0} e^{-t} .
$$

More importantly, the solution $x(t)$ of the last equation of the differential system

$$
x(t)=x_{0} e^{k\left(-\frac{1}{2} e^{-t}\left(2\left(p_{0}+s_{0}\right)+\sqrt{2 \pi} s_{0} \operatorname{Erf}\left(\frac{t}{\sqrt{2}}\right)\right)+p_{0}+s_{0}\right)},
$$

generates a new growth model not encountered to have been described in the literature.

We illustrate our new model for fixed $s_{0}, p_{0}$ and various parameters $k$ and $x_{0}$ (Fig. 1).

Remark. It is important to study the characteristic - "super saturation" of the model to the horizontal asymptote.

In this connection, the following Proposition is valid. 
Proposition. For the new model (8) it is fulfilled that

$$
\lim _{t \rightarrow \infty} x(t)=x_{0} e^{k\left(s_{0}+p_{0}\right)} \text {. }
$$
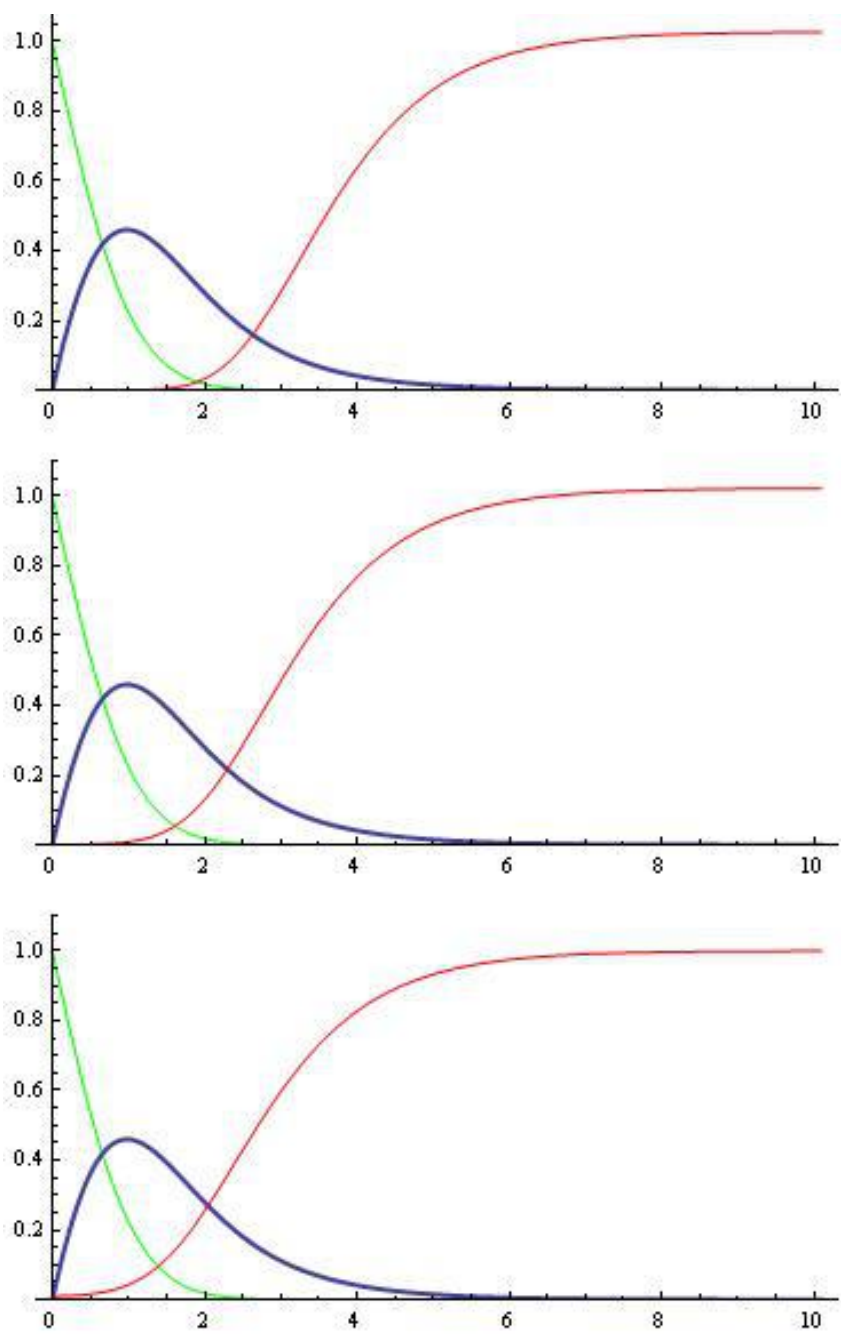

Fig. 1. The functions $x\left(t\right.$ ) (red), $p\left(t\right.$ ) (blue), $s\left(t\right.$ ) (green) for fixed $s_{0}=1, p_{0}=0$ :

a) $k=11.54, x_{0}=0.00001$; b) $k=6.93, x_{0}=0.001$; c) $k=4.605, x_{0}=0.01$

\subsection{Some applications}

The consideration of such a dynamic model in the present article is dictated by our passionate desire to offer an adequate model with which to well approximate some specific data (including datasets from computer viruses propagation), characterized by rapid growth in the initial time interval. 
Example 1. We consider the following specific data [12]:

data_CDF_1: $=\{\{1,0.6762\},\{2,0.8286\},\{3,0.8667\},\{4,0.9143\}$,

$\{5,0.9333\},\{6,0.9429\},\{7,0.9524\},\{8,0.9571\},\{9,0.9667\}$,

$\{10,0.9714\},\{11,0.9733\},\{14,0.9810\},\{20,0.9829\},\{23,0.9857\}$,

$\{25,0.9885\},\{55,0.9905\}\}$.

The model (8) for $p_{0}=0, s_{0}=1, x_{0}=0.5, k=0.660972$ is visualized on Fig. 2.

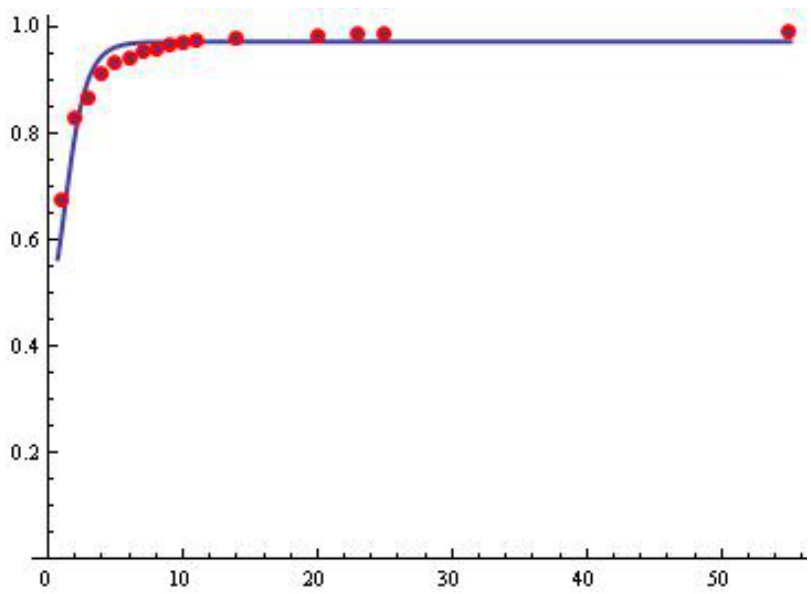

Fig. 2. The fitted model (8) for approximation of the data: "data_CDF_1" [12]

Example 2. Storm worm was one of the most biggest cyber threats of 2008 [13]. We consider the following data:

data_Storm_IDs

$:=\{\{1,0.843\},\{4,0.926\},\{5,0.954\},\{6,0.967\},\{7,0.976\}$,

$\{8,0.981\},\{9,0.985\},\{10,0.991\},\{22,0.995\},\{38,0.997\},\{51,0.998\}$,

$\{64,0.9985\},\{74,0.999\},\{83,1\},\{100,1\}\}$.

The model (8) for $p_{0}=0, s_{0}=1, x_{0}=0.5, k=0.683003$ is visualized on Fig. 3.

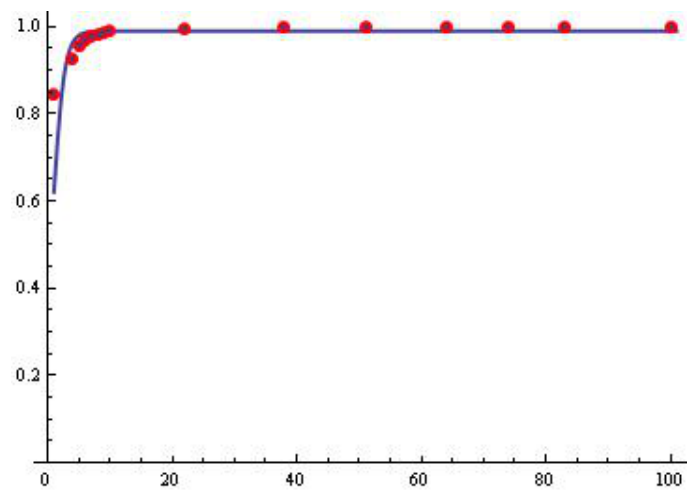

Fig. 3. The fitted model (8) for approximation of the data "data-Storm" 
Example 3. For the data (collected from the 140 telecommunication systems that manage the radio access part of wireless systems by week (see, for example [14])) the model (8) for

$$
k=3.44078, s_{0}=1, p_{0}=0.65, x_{0}=0.17
$$

is depicted on Fig. 4.

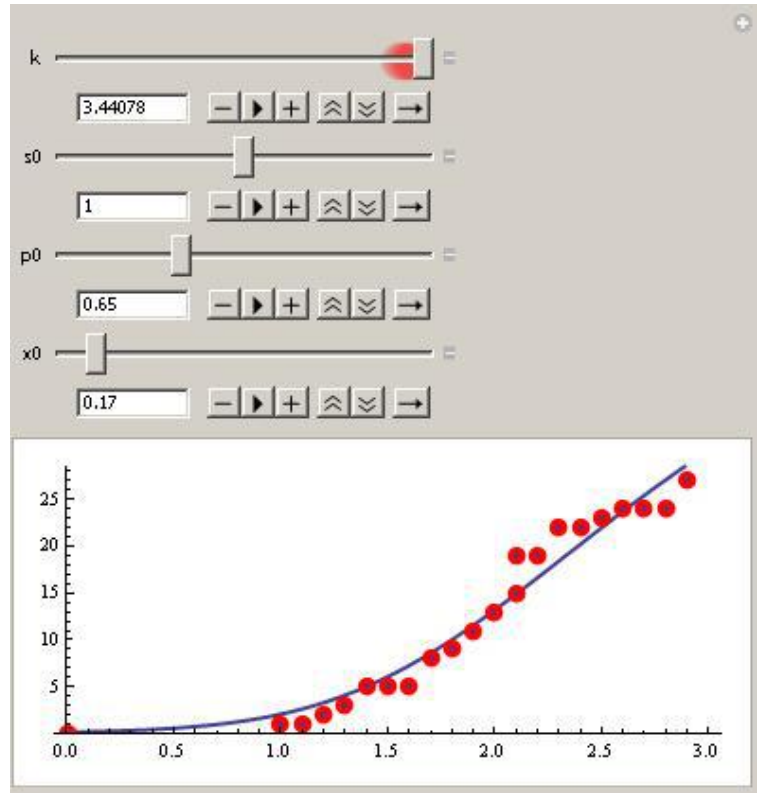

Fig. 4. The model (8) (Example 3)

Example 4. The mean value functions of nine models for the "Failure data" from the Debugging Theory are considered by Lee, Chang and Pham [14], (Fig. 5)

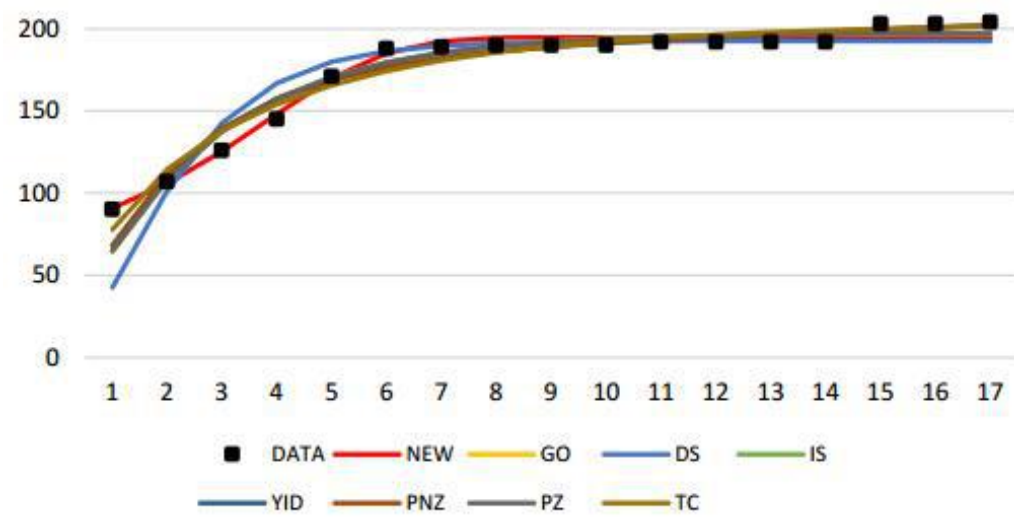

Fig. 5. The mean value functions of nine models (including GO (Goel-Okumoto), NEW (Le-ChangPham), DS (Delayed S-shaped), PZ (Pham-Zhang) and other models for this data [14]) 
For the data from [14] the model (8) for $k=14.3787, s_{0}=-0.521269$, $p_{0}=1.1, x_{0}=0.0441936$, is depicted on Fig. 6 . (We have adopted a scale on the horizontal axis: 0.1 division corresponds to one time interval).

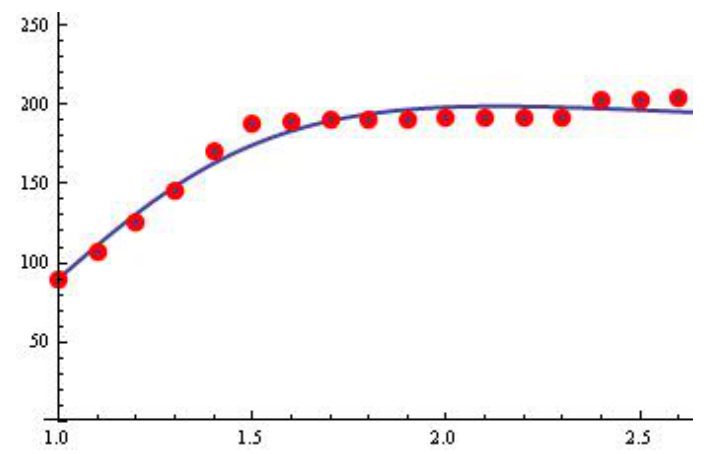

Fig. 6. The model (8) for the "Failure data"

The experiments show that in some cases the use of the growth model proposed in this article is satisfactory.

Specialists working in this scientific field have a say.

For other results, see [15-19].

Acknowledgments: This work has been accomplished with the financial support by the Grant No BG05M2OP001-1.001-0003, financed by the Science and Education for Smart Growth Operational Program (2014-2020) and co-financed by the European Union through the European structural and Investment funds.

\section{References}

1. K y u r k c h i e v, N. On a Sigmoidal Growth Function Generated by Reaction Networks. - Some Extensions and Applications, Communications in Applied Analysis, Vol. 23, 2019, No 3, pp. 383-400.

2. Marko v, S. Reaction Networks Reveal New Links between Gompertz and Verhulst Growth Functions. - Biomath, Vol. 8, 2019, No 1.

3. M a r k o v, S. On a Class of Generalized Gompertz-Bateman Growth-Decay Models. - Biomath Communications, Vol. 6, 2019, No 1, pp. 51-64.

4. K y u r k c h i e v, N. Some New Classes of Growth Functions Generated by Reaction Networks and Based on "Correcting Amendments" of Bateman-Gompertz and Bateman-GompertzMakeham-Type. I. - Communications in Applied Analysis, Vol. 24, 2020, No 1, pp. 13-29.

5. K y u r k c h i e v, N. On a Class of Growth Curves with Exponentially Variable Transfer Generated by Reaction Networks. II. - International Electronic Journal of Pure and Applied Mathematics, Vol. 14, 2020, No 1, pp. 21-29.

6. B a t e m a n, H. The Solution of a System of Differential Equations Occurring in the Theory of Radio-Active Transformations. - Proc. of Cambridge Philosophical Society, Vol. 15, 1910, pp. 423-427.

7. Go m p e r t z, B. On the Nature of the Function Expressive of the Law of Human Mortality, and on a New Mode of Determining the 62 Value of Life Contingencies. - In: Philosophical Transactions of the Royal Society of London. Vol. 115. 1825, pp. 513-583.

8. Rogers-Bennet t, L., D. W. Rogers. A Two-Step Growth Curve: Approach to the von Bertalanffy and Gompertz Equations. - Advances in Pure Mathematics, Vol. 6, 2016, pp. 321-330. 
9. B a n k s, R. Growth and Diffusion Phenomena. - Mathematical Frameworks and Applications, Springer-Verlag, 1994.

10. Le n t e, G. Deterministic Kinetics in Chemistry and Systems Biology. - In: Briefs in Molecular Science, Cham Heidelberg, New York, Dordrecht, London, Springer, 2015.

11. M u r r a y, J. D. Mathematical Biology: I. An Introduction. 3rd Ed. New York, Berlin, Heidelberg, Springer-Verlag, 2002.

12. C o n t i, M., A. Ga n g w a 1, S. R u j. On the Economic Significance of Ransomware Campaigns: A Bitcoin Transactions Perspective. - Computers \& Security, Vol. 79, 2018, pp. 162-189.

13. S a r a t, S., A. Terzi s. HiNRG Technical Report. 01-10-2007, Measuring the Storm Worm Network, 2007.

14. L e e, D., I. C h a n g, H. P h a m. Software Reliability Model with Dependent Failures and SPRT. Mathematics, Vol. 8, 2020, No 8. 15 p.

15. M a r k o v, S., A. I 1 i e v, A. R a h n e v, N. K y u r k c h i e v. A Note on the $n$-Stage Growth Model. Overview. - Biomath Communications, Vol. 5, 2018, No 2, pp. 79-100.

16. A n gue lov, R., M. B or is ov, A. Ili e v, N. K y u rk chi e v, S. M arkov. On the Chemical Meaning of Some Growth Models Possessing Gompertzian-Type Property. - Math. Meth. Appl. Sci., 2017, pp. 1-12.

17. K y u r k c h i e v, N., V. K y u r k c h i e v, A. I 1 i e v, A. R a h n e v. Some Nonstandard Differential Models with Applications to the Population Dynamics and Computer Viruses Propagation. Dynamic Systems and Applications, Vol. 28, 2019, No 3, pp. 757-788.

18. K y u r k c h i e v, N. Investigations on a Hyper-Logistic Model. Some Applications. - Dynamic Systems and Applications, Vol. 28, 2019, No 2, pp. 351-369.

19. K y u r k c h i e v, N. Selected Topics in Mathematical Modeling: Some New Trends (Dedicated to Academician Blagovest Sendov (1932-2020)). LAP LAMBERT Academic Publishing, 2020. ISBN: 978-620-2-51403-3.

Received: 05.09.2020; Second Version: 23.10.2020; Accepted: 30.10 .2020 